\title{
PSEUDOSTARLIKE AND PSEUDOCONVEX SOLUTIONS OF A DIFFERENTIAL EQUATION WITH EXPONENTIAL COEFFICIENTS
}

M. M. Sheremeta. Pseudostarlike and pseudoconvex solutions of a differential equation with exponential coefficients, Mat. Stud. 56 (2021), 39-47.

Dirichlet series $F(s)=e^{s}+\sum_{k=1}^{\infty} f_{k} e^{s \lambda_{k}}$ with the exponents $1<\lambda_{k} \uparrow+\infty$ and the abscissa of absolute convergence $\sigma_{a}[F] \geq 0$ is said to be pseudostarlike of order $\alpha \in[0,1)$ and type $\beta \in(0,1]$ if

$$
\left|\frac{F^{\prime}(s)}{F(s)}-1\right|<\beta\left|\frac{F^{\prime}(s)}{F(s)}-(2 \alpha-1)\right| \text { for all } s \in \Pi_{0}=\{s: \operatorname{Re} s<0\} .
$$

Similarly, the function $F$ is said to be pseudoconvex of order $\alpha \in[0,1)$ and type $\beta \in(0,1]$ if

$$
\left|\frac{F^{\prime \prime}(s)}{F^{\prime}(s)}-1\right|<\beta\left|\frac{F^{\prime \prime}(s)}{F^{\prime}(s)}-(2 \alpha-1)\right| \text { for all } s \in \Pi_{0} .
$$

Some conditions are found on the parameters $b_{0}, b_{1}, c_{0}, c_{1}, c_{2}$ and the coefficients $a_{n}$, under which the differential equation

$$
\frac{d^{2} w}{d s^{2}}+\left(b_{0} e^{s}+b_{1}\right) \frac{d w}{d s}+\left(c_{0} e^{2 s}+c_{1} e^{s}+c_{2}\right) w=\sum_{n=1}^{\infty} a_{n} e^{n s}
$$

has an entire solution which is pseudostarlike or pseudoconvex of order $\alpha \in[0,1)$ and type $\beta \in(0,1]$. It is proved that by some conditions for such solution the asymptotic equality holds

$$
\ln \max \{|F(\sigma+i t)|: t \in \mathbb{R}\}=\frac{1+o(1)}{2}\left(\left|b_{0}\right|+\sqrt{\left|b_{0}\right|^{2}+4\left|c_{0}\right|}\right) \text { as } \sigma \rightarrow+\infty \text {. }
$$

1. Introduction and auxiliary results. An analytic univalent in $\mathbb{D}=\{z:|z|<1\}$ function $f(z)=\sum_{n=0}^{\infty} f_{n} z^{n}$ is said to be convex if $f(\mathbb{D})$ is a convex domain. It is well known $\left[1\right.$, p. 203] that the condition $\operatorname{Re}\left\{1+z f^{\prime \prime}(z) / f^{\prime}(z)\right\}>0(z \in \mathbb{D})$ is necessary and sufficient for the convexity of $f$. By W. Kaplan [2] the function $f$ is said to be close-to-convex in $\mathbb{D}$ (see also $\left[1\right.$, p. 583]) if there exists a convex in $\mathbb{D}$ function $\Phi$ such that $\operatorname{Re}\left(f^{\prime}(z) / \Phi^{\prime}(z)\right)>0(z \in \mathbb{D})$. Close-to-convex function $f$ has a characteristic property that the complement $G$ of the domain $f(\mathbb{D})$ can be filled with rays $L$ which go from $\partial G$ and lie in $G$. Every close-to-convex in $\mathbb{D}$ function $f$ is univalent in $\mathbb{D}$ and, therefore, $f^{\prime}(0) \neq 0$. Hence, it follows that the function $f$ is close-to-convex in $\mathbb{D}$ if and only if the function $g(z)=z+\sum_{n=2}^{\infty} g_{n} z^{n}$ is close-to-convex in $\mathbb{D}$, where $g_{n}=f_{n} / f_{1}$. Such function $g$ is said to be starlike if $f(\mathbb{D})$ is a starlike domain. It is well known [1, p. 203] that the condition $\operatorname{Re}\left\{z f^{\prime}(z) / f(z)\right\}>0(z \in \mathbb{D})$ is necessary and sufficient for the starlikeness of $g$.

S.M. Shah [3] indicated conditions on real parameters $\beta_{0}, \beta_{1}, \gamma_{0}, \gamma_{1}, \gamma_{2}$ of the differential equation $z^{2} w^{\prime \prime}+\left(\beta_{0} z^{2}+\beta_{1} z\right) w^{\prime}+\left(\gamma_{0} z^{2}+\gamma_{1} z+\gamma_{2}\right) w=0$, under which there exists an entire transcendental solution $f$ such that $f$ and all its derivatives are close-to-convex in $\mathbb{D}$. The

2010 Mathematics Subject Classification: 30B10, 30B50, 30C45, 35 B08.

Keywords: linear differential equation; Dirichlet series; pseudostarlikeness; pseudoconvexity. doi:10.30970/ms.56.1.39-47 
investigations are continued in the papers $[4-9]$. In $[10,11]$ it is studied the closeness-toconvexity of the second order non-homogeneous linear differential equation

$$
z^{2} w^{\prime \prime}+\left(\beta_{0} z^{2}+\beta_{1} z\right) w^{\prime}+\left(\gamma_{0} z^{2}+\gamma_{1} z+\gamma_{2}\right) w=A(z),
$$

where $A(z)=\sum_{n=1}^{\infty} a_{n} z^{n}$ and radius of convergence of the last power series is $R[A] \geq 1$. Substituting $z=e^{s}$ we obtain the differential equation

$$
\frac{d^{2} w}{d s^{2}}+\left(b_{0} e^{s}+b_{1}\right) \frac{d w}{d s}+\left(c_{0} e^{2 s}+c_{1} e^{s}+c_{2}\right) w=\sum_{n=1}^{\infty} a_{n} e^{n s}
$$

where $b_{0}=\beta_{0}, b_{1}=\beta_{1}-1, c_{j}=\gamma_{j}$ and Dirichlet series $\sum_{n=1}^{\infty} a_{n} e^{n s}$ is absolutely convergent in a half-plane $\{s: \operatorname{Re} s<a\}$ with $a \geq 0$.

Now, let $\Lambda=\left(\lambda_{k}\right)$ be an increasing to $+\infty$ sequence of positive numbers $\left(\lambda_{1}>1\right)$ and $S D(\Lambda, 0)$ be a class of Dirichlet series

$$
F(s)=e^{s}+\sum_{k=1}^{\infty} f_{k} \exp \left\{s \lambda_{k}\right\}, \quad f_{k} \neq 0, \quad s=\sigma+i t
$$

with the exponents $\Lambda$ and the abscissa of absolute convergence $\sigma_{a}[F]=0$. It is known [12] (see also [13, p. 135] that each function $F \in S D(\Lambda, 0)$ is non-univalent in $\Pi_{0}=\{s: \operatorname{Re} s<0\}$, but there exist conformal in $\Pi_{0}$ functions (2), and if $\sum_{k=1}^{\infty} \lambda_{k}\left|f_{k}\right| \leq 1$ then function (2) is conformal in $\Pi_{0}$. A conformal function (2) in $\Pi_{0}$ is said to be pseudostarlike if $\operatorname{Re}\left\{F^{\prime}(s) / F(s)\right\}>0$ for $s \in \Pi_{0}$. In [12] (see also [13, p. 139]) it is proved that if $\sum_{k=1}^{\infty} \lambda_{k}\left|f_{k}\right| \leq 1$ then function (2) is pseudostarlike.

A conformal function (2) in $\Pi_{0}$ is said to be pseudostarlike of order $\alpha$ if

$$
\operatorname{Re}\left\{F^{\prime}(s) / F(s)\right\}>\alpha \in[0,1), \quad s \in \Pi_{0} .
$$

Since the inequality $|w-1|<|w-(2 \alpha-1)|$ holds if and only if $\operatorname{Re} w>\alpha$, function (3) is pseudostarlike of the order $\alpha$ if and only if

$$
\left|\frac{F^{\prime}(s)}{F(s)}-1\right|<\left|\frac{F^{\prime}(s)}{F(s)}-(2 \alpha-1)\right| \text { for } s \in \Pi_{0} \text {. }
$$

Therefore, as in [14] the conformal function (2) in $\Pi_{0}$ is called pseudostarlike of order $\alpha \in$ $[0,1)$ and type $\beta \in(0,1]$ if

$$
\left|\frac{F^{\prime}(s)}{F(s)}-1\right|<\beta\left|\frac{F^{\prime}(s)}{F(s)}-(2 \alpha-1)\right|, \quad s \in \Pi_{0} .
$$

Lemma 1 ([14]). If

$$
\sum_{k=1}^{\infty}\left\{(1+\beta) \lambda_{k}-\beta(2 \alpha-1)-1\right\}\left|f_{k}\right| \leq 2 \beta(1-\alpha)
$$

then (2) is pseudostarlike of order $\alpha$ and type $\beta$. 
Similarly, a conformal function (2) in $\Pi_{0}$ is said to be pseudoconvex if

$$
\operatorname{Re}\left\{F^{\prime \prime}(s) / F^{\prime}(s)\right\}>0 \text { for } s \in \Pi_{0} \text {. }
$$

In [12] and [13, p. 139] it is proved that if

$$
\sum_{k=1}^{\infty} \lambda_{k}^{2}\left|f_{k}\right| \leq 1
$$

then function (2) is pseudoconvex. Here we call the function (2) pseudoconvex of the order $\alpha \in[0,1)$ if $\operatorname{Re}\left\{F^{\prime \prime}(s) / F^{\prime}(s)\right\}>\alpha$, and pseudoconvex of order $\alpha$ and type $\beta \in(0,1]$ if [14]

$$
\left|\frac{F^{\prime \prime}(s)}{F^{\prime}(s)}-1\right|<\beta\left|\frac{F^{\prime \prime}(s)}{F^{\prime}(s)}-(2 \alpha-1)\right|, \quad s \in \Pi_{0} .
$$

Since $F^{\prime \prime}(s) / F^{\prime}(s)=G^{\prime}(s) / G(s)$, where $G(s)=e^{s}+\sum_{k=1}^{\infty} g_{k} \exp \left\{s \lambda_{k}\right\}$ and $g_{k}=\lambda_{k} f_{k}$, the function $F$ is pseudoconvex of order $\alpha \in[0,1)$ and type $\beta \in(0,1]$ if and only if the function $G$ is pseudostarlike of order $\alpha \in[0,1)$ and type $\beta \in(0,1]$. Therefore, from Lemma 1 one can easily obtain the corresponding result for pseudoconvex functions.

Lemma 2 ([14]). If

$$
\sum_{k=1}^{\infty} \lambda_{k}\left\{(1+\beta) \lambda_{k}-\beta(2 \alpha-1)-1\right\}\left|f_{k}\right| \leq 2 \beta(1-\alpha)
$$

then (2) is pseudoconvex of order $\alpha$ and type $\beta$.

Here we investigate the conditions under which equation (1) has solutions that are pseudostarlike or pseudoconvex of order $\alpha$ and type $\beta$. We remark that if $b_{0}=b_{1}=0$ then such a problem is solved in [12-13] for the case of $\alpha=0, \beta=1$.

2. Recurrent formulas. Suppose that Dirichlet series (2) satisfies (1). Then

$$
\begin{gathered}
\left(1+b_{1}+c_{2}\right) e^{s}+\left(b_{0}+c_{1}\right) e^{2 s}++c_{0} e^{3 s}+\sum_{k=1}^{\infty}\left(\lambda_{k}^{2}+b_{1} \lambda_{k}+c_{2}\right) f_{k} \exp \left\{s \lambda_{k}\right\}+ \\
+\sum_{k=1}^{\infty}\left(b_{0} \lambda_{k}+c_{1}\right) f_{k} \exp \left\{s\left(\lambda_{k}+1\right)\right\}+\sum_{k=1}^{\infty} c_{0} f_{k} \exp \left\{s\left(\lambda_{k}+2\right)\right\}=a_{1} e^{s}+\sum_{n=2}^{\infty} a_{n} e^{n s}
\end{gathered}
$$

Since $\lambda_{1}>1$, hence as $s \rightarrow-\infty$ we have $\left(1+b_{1}+c_{2}\right) e^{s}=(1+o(1)) a_{1} e^{s}$, i. e. $1+b_{1}+c_{2}=a_{1}$. Therefore, (6) implies

$$
\begin{aligned}
& \left(b_{0}+c_{1}\right) e^{2 s}+c_{0} e^{3 s}+\left(\lambda_{1}^{2}+b_{1} \lambda_{1}+c_{2}\right) f_{1} \exp \left\{s \lambda_{1}\right\}+\sum_{k=2}^{\infty}\left(\lambda_{k}^{2}+b_{1} \lambda_{k}+c_{2}\right) f_{k} \exp \left\{s \lambda_{k}\right\}+ \\
& +\sum_{k=1}^{\infty}\left(b_{0} \lambda_{k}+c_{1}\right) f_{k} \exp \left\{s\left(\lambda_{k}+1\right)\right\}+\sum_{k=1}^{\infty} c_{0} f_{k} \exp \left\{s\left(\lambda_{k}+2\right)\right\}=a_{2} e^{2 s}+\sum_{n=3}^{\infty} a_{n} e^{n s} .
\end{aligned}
$$

Since $\lambda_{1}+1>1$ and $\lambda_{2}>\lambda_{1}$, hence as $s \rightarrow-\infty$ we have

$$
\left(\lambda_{1}^{2}+b_{1} \lambda_{1}+c_{2}\right) f_{1} \exp \left\{s \lambda_{1}\right\}+o\left(e^{s \lambda_{1}}\right)=\left(a_{2}-b_{0}-c_{1}\right) e^{2 s}+o\left(e^{2 s}\right) .
$$


Therefore, if $\lambda_{1}^{2}+b_{1} \lambda_{1}+c_{2} \neq 0$ then $\lambda_{1}=2$ and

$$
f_{1}=\frac{a_{2}-b_{0}-c_{1}}{4+2 b_{1}+c_{2}}
$$

Therefore, (7) implies

$$
\begin{gathered}
c_{0} e^{3 s}+\left(\lambda_{2}^{2}+b_{1} \lambda_{2}+c_{2}\right) f_{2} \exp \left\{s \lambda_{2}\right\}+\sum_{k=3}^{\infty}\left(\lambda_{k}^{2}+b_{1} \lambda_{k}+c_{2}\right) f_{k} \exp \left\{s \lambda_{k}\right\}+ \\
+\left(2 b_{0}+c_{1}\right) f_{1} \exp \{3 s\}+\sum_{k=2}^{\infty}\left(b_{0} \lambda_{k}+c_{1}\right) f_{k} \exp \left\{s\left(\lambda_{k}+1\right)\right\}+ \\
+\sum_{k=1}^{\infty} c_{0} f_{k} \exp \left\{s\left(\lambda_{k}+2\right)\right\}=a_{3} e^{3 s}+\sum_{n=4}^{\infty} a_{n} e^{n s} .
\end{gathered}
$$

Hence, it follows that

$$
\left(\lambda_{2}^{2}+b_{1} \lambda_{2}+c_{2}\right) f_{2} \exp \left\{s \lambda_{2}\right\}+o\left(e^{s \lambda_{2}}\right)=\left(a_{3}-c_{0}-\left(2 b_{0}+c_{1}\right) f_{1}\right) e^{3 s}+o\left(e^{3 s}\right),
$$

as $s \rightarrow-\infty$ and if $\lambda_{2}^{2}+b_{1} \lambda_{2}+c_{2} \neq 0$ then $\lambda_{2}=3$ and

$$
f_{2}=\frac{a_{3}-c_{0}}{9+3 b_{1}+c_{2}}-\frac{2 b_{0}+c_{1}}{9+3 b_{1}+c_{2}} f_{1} .
$$

Therefore, (9) implies

$$
\begin{aligned}
\left(\lambda_{3}^{2}+\right. & \left.b_{1} \lambda_{3}+c_{2}\right) f_{3} \exp \left\{s \lambda_{3}\right\}+\sum_{k=4}^{\infty}\left(\lambda_{k}^{2}+b_{1} \lambda_{k}+c_{2}\right) f_{k} \exp \left\{s \lambda_{k}\right\}+ \\
& +\left(3 b_{0}+c_{1}\right) f_{2} e^{4 s}+\sum_{k=3}^{\infty}\left(b_{0} \lambda_{k}+c_{1}\right) f_{k} \exp \left\{s\left(\lambda_{k}+1\right)\right\}+ \\
& +c_{0} f_{1} e^{4 s}+\sum_{k=2}^{\infty} c_{0} f_{k} \exp \left\{s\left(\lambda_{k}+2\right)\right\}=a_{4} e^{4 s}+\sum_{n=5}^{\infty} a_{n} e^{n s}
\end{aligned}
$$

Hence, it follows that

$$
\left(\lambda_{3}^{2}+b_{1} \lambda_{3}+c_{2}\right) f_{3} \exp \left\{s \lambda_{3}\right\}+o\left(e^{s \lambda_{3}}\right)=\left(a_{4}-c_{0} f_{1}-\left(3 b_{0}+c_{1}\right) f_{2}\right) e^{3 s}+o\left(e^{3 s}\right),
$$

as $s \rightarrow-\infty$ and if $\lambda_{3}^{2}+b_{1} \lambda_{3}+c_{2} \neq 0$ then $\lambda_{3}=4$ and

$$
f_{3}=\frac{a_{4}}{16+4 b_{1}+c_{2}}-\frac{3 b_{0}+c_{1}}{16+4 b_{1}+c_{2}} f_{2}-\frac{c_{0}}{16+4 b_{1}+c_{2}} f_{1} .
$$

Therefore, (11) implies

$$
\begin{gathered}
\left(\lambda_{4}^{2}+b_{1} \lambda_{4}+c_{2}\right) f_{4} \exp \left\{s \lambda_{4}\right\}+\sum_{k=5}^{\infty}\left(\lambda_{k}^{2}+b_{1} \lambda_{k}+c_{2}\right) f_{k} \exp \left\{s \lambda_{k}\right\}+ \\
+\left(4 b_{0}+c_{1}\right) f_{3} e^{5 s}+\sum_{k=4}^{\infty}\left(b_{0} \lambda_{k}+c_{1}\right) f_{k} \exp \left\{s\left(\lambda_{k}+1\right)\right\}+
\end{gathered}
$$




$$
+c_{0} f_{2} e^{5 s}+\sum_{k=3}^{\infty} c_{0} f_{k} \exp \left\{s\left(\lambda_{k}+2\right)\right\}=a_{5} e^{5 s}+\sum_{n=6}^{\infty} a_{n} e^{n s},
$$

whence as above it follows that if $\lambda_{4}^{2}+b_{1} \lambda_{4}+c_{2} \neq 0$ then $\lambda_{4}=5$ and

$$
f_{4}=\frac{a_{5}}{25+5 b_{1}+c_{2}}-\frac{4 b_{0}+c_{1}}{25+5 b_{1}+c_{2}} f_{3}-\frac{c_{0}}{25+5 b_{1}+c_{2}} f_{2} .
$$

Continuing this process, we will come to the formulas $\lambda_{k}=k+1$ and

$$
\begin{gathered}
f_{k}=\frac{a_{k+1}}{(k+1)^{2}+(k+1) b_{1}+c_{2}}- \\
-\frac{k b_{0}+c_{1}}{(k+1)^{2}+(k+1) b_{1}+c_{2}} f_{k-1}-\frac{c_{0}}{(k+1)^{2}+(k+1) b_{1}+c_{2}} f_{k-2}
\end{gathered}
$$

for $k \geq 3$, provided $\lambda_{k}^{2}+b_{1} \lambda_{k}+c_{2} \neq 0$.

Thus, the following statement is correct.

Lemma 3. If $1+b_{1}+c_{2}=a_{1}$ and $k^{2}+k b_{1}+c_{2} \neq 0$ for all $k \geq 2$ then differential equation (1) has the solution

$$
F(s)=e^{s}+\sum_{k=1}^{\infty} f_{k} \exp \{s(k+1)\},
$$

where the coefficients $f_{1}$ and $f_{2}$ are defined by formulas (8) and (10), and for $k \geq 3$ recurrent formula (13) is true.

2. Pseudostarlikeness. At the first, we remark that for function (14) condition (5) has the form

$$
\sum_{k=1}^{\infty} B_{k}\left|f_{k}\right| \leq 2 \beta(1-\alpha), \quad B_{k}=(1+\beta) k+2 \beta(1-\alpha) .
$$

We put $A_{k}=(k+1)^{2}+(k+1) b_{1}+c_{2}$ and suppose that $b_{1} \geq 0$ and $c_{2} \geq 0$. Then $A_{k}>0$ for all $k \geq 1$ and from (8), (10) and (13) we get $f_{1}=\frac{a_{2}-b_{0}-c_{1}}{A_{1}}, f_{2}=\frac{a_{3}-c_{0}}{A_{2}}-\frac{2 b_{0}+c_{1}}{A_{2}} f_{1}$ and $f_{k}=\frac{a_{k+1}}{A_{k}}-\frac{k b_{0}+c_{1}}{A_{k}} f_{k-1}-\frac{c_{0}}{A_{k}} f_{k-2}$ for $k \geq 3$. Therefore,

$$
\begin{gathered}
\sum_{k=1}^{\infty} B_{k}\left|f_{k}\right| \leq B_{1}\left|f_{1}\right|+B_{2}\left|f_{2}\right|+\sum_{k=1}^{\infty} B_{k}\left|f_{k}\right| \leq \\
\leq B_{1}\left|f_{1}\right|+B_{2}\left|f_{2}\right|+\sum_{k=3}^{\infty} B_{k} \frac{\left|a_{k+1}\right|}{A_{k}}+\sum_{k=3}^{\infty} B_{k} \frac{k\left|b_{0}\right|+\left|c_{1}\right|}{A_{k}}\left|f_{k-1}\right|+\sum_{k=3}^{\infty} B_{k} \frac{\left|c_{0}\right|}{A_{k}}\left|f_{k-2}\right|= \\
=B_{1}\left|f_{1}\right|+B_{2}\left|f_{2}\right|+\sum_{k=3}^{\infty} B_{k} \frac{\left|a_{k+1}\right|}{A_{k}}+\sum_{k=2}^{\infty} B_{k+1} \frac{(k+1)\left|b_{0}\right|+\left|c_{1}\right|}{A_{k+1}}\left|f_{k}\right|+\sum_{k=1}^{\infty} B_{k+2} \frac{\left|c_{0}\right|}{A_{k+2}}\left|f_{k}\right|= \\
=B_{1}\left|f_{1}\right|+B_{2}\left|f_{2}\right|+\sum_{k=3}^{\infty} B_{k} \frac{\left|a_{k+1}\right|}{A_{k}}-B_{2} \frac{|2| b_{0}\left|+c_{1}\right|}{A_{2}}\left|f_{1}\right|+ \\
+\sum_{k=1}^{\infty}\left(B_{k+1} \frac{(k+1)\left|b_{0}\right|+\left|c_{1}\right|}{A_{k+1}}+B_{k+2} \frac{\left|c_{0}\right|}{A_{k+2}}\right)\left|f_{k}\right| .
\end{gathered}
$$


Since $B_{2}\left|f_{2}\right|-B_{2} \frac{2\left|b_{0}\right|+\left|c_{1}\right|}{A_{2}}\left|f_{1}\right| \leq B_{2} \frac{\left|a_{3}-c_{0}\right|}{A_{2}}$, hence we obtain

$$
\begin{gathered}
\sum_{k=1}^{\infty} B_{k}\left|f_{k}\right| \leq B_{1} \frac{\left|a_{2}-b_{0}-c_{1}\right|}{A_{1}}+B_{2} \frac{\left|a_{3}-c_{0}\right|}{A_{2}}+\sum_{k=3}^{\infty} B_{k} \frac{\left|a_{k+1}\right|}{A_{k}}+ \\
+\sum_{k=1}^{\infty}\left(\frac{B_{k+1}}{B_{k}} \frac{(k+1)\left|b_{0}\right|+\left|c_{1}\right|}{A_{k+1}}+\frac{B_{k+2}}{B_{k}} \frac{\left|c_{0}\right|}{A_{k+2}}\right) B_{k}\left|f_{k}\right| .
\end{gathered}
$$

If we put

$$
\eta=\frac{2(1+\beta)+2 \beta(1-\alpha)}{1+\beta+2 \beta(1-\alpha)} \frac{3\left|b_{0}\right|+\left|c_{1}\right|+2\left|c_{0}\right|}{9+3 b_{1}}
$$

and

$$
Q=\frac{1+\beta+2 \beta(1-\alpha)}{4+2 b_{1}+c_{2}}\left(\left|a_{2}-b_{0}-c_{1}\right|+2\left|a_{3}-c_{0}\right|\right)+\sum_{k=3}^{\infty} \frac{(1+\beta) k+2 \beta(1-\alpha)}{(k+1)^{2}+(k+1) b_{1}+c_{2}}\left|a_{k+1}\right|
$$

then using (16) we can prove the following theorem.

Theorem 1. Let $b_{1} \geq 0, c_{2} \geq 0$ and $a_{1}=1+b_{1}+c_{2}$. If

$$
Q \leq 2 \beta(1-\eta)(1-\alpha)
$$

then differential equation (1) has solution (14) which is pseudostarlike in $\Pi_{0}$ of order $\alpha$ and type $\beta$.

Proof. The conditions $b_{1} \geq 0$ and $c_{2} \geq 0$ imply $k^{2}+k b_{1}+c_{2} \neq 0$ for all $k \geq 2$ and, thus, the conditions of Lemma 3 are valid.

Since $\frac{B_{k+1}}{B_{k}}=1+\frac{1+\beta}{(1+\beta) k+2 \beta(1-\alpha)} \downarrow 1$ as $k \rightarrow \infty$, we have $\frac{B_{k+1}}{B_{k}} \leq \frac{B_{2}}{B_{1}}$ and $\frac{B_{k+2}}{B_{k}} \leq \frac{B_{3}}{B_{1}}$ for all $k \geq 1$. Also, for $k \geq 1$

$$
\begin{gathered}
\frac{(k+1)\left|b_{0}\right|+\left|c_{1}\right|}{A_{k+1}}=\frac{(k+1)\left|b_{0}\right|+\left|c_{1}\right|}{(k+2)^{2}+(k+2) b_{1}+c_{2}} \leq \frac{(k+1)\left|b_{0}\right|+\left|c_{1}\right|}{(k+2)^{2}+(k+2) b_{1}} \leq \\
\leq \frac{(k+2)\left|b_{0}\right|}{(k+2)^{2}+(k+2) b_{1}}+\frac{\left|c_{1}\right|}{(k+2)^{2}+(k+2) b_{1}} \leq \frac{\left|b_{0}\right|}{3+b_{1}}+\frac{\left|c_{1}\right|}{9+3 b_{1}}=\frac{3\left|b_{0}\right|+\left|c_{1}\right|}{9+3 b_{1}}
\end{gathered}
$$

and

$$
\frac{\left|c_{0}\right|}{A_{k+2}}=\frac{\left|c_{0}\right|}{(k+3)^{2}+(k+3) b_{1}+c_{2}} \leq \frac{\left|c_{0}\right|}{(k+3)^{2}+(k+3) b_{1}} \leq \frac{\left|c_{0}\right|}{16+4 b_{1}} .
$$

Thus,

$$
\begin{gathered}
\frac{B_{k+1}}{B_{k}} \frac{(k+1)\left|b_{0}\right|+\left|c_{1}\right|}{A_{k+1}}+\frac{B_{k+2}}{B_{k}} \frac{\left|c_{0}\right|}{A_{k+2}} \leq \\
\leq \frac{2(1+\beta)+2 \beta(1-\alpha)}{1+\beta+2 \beta(1-\alpha)} \frac{3\left|b_{0}\right|+\left|c_{1}\right|}{9+3 b_{1}}+\frac{3(1+\beta)+2 \beta(1-\alpha)}{1+\beta+2 \beta(1-\alpha)} \frac{\left|c_{0}\right|}{16+4 b_{1}}
\end{gathered}
$$

and, since $\frac{3(1+\beta)+2 \beta(1-\alpha)}{16+4 b_{1}} \leq 2 \frac{2(1+\beta)+2 \beta(1-\alpha)}{9+3 b_{1}}$, we have

$$
\frac{B_{k+1}}{B_{k}} \frac{(k+1)\left|b_{0}\right|+\left|c_{1}\right|}{A_{k+1}}+\frac{B_{k+2}}{B_{k}} \frac{\left|c_{0}\right|}{A_{k+2}} \leq \eta .
$$


Since $\frac{B_{2}}{A_{2}}=\frac{2(1+\beta)++2 \beta(1-\alpha)}{9+3 b_{1}+c_{2}} \leq 2 \frac{B_{1}}{A_{1}}=2 \frac{1+\beta+2 \beta(1-\alpha)}{4+2 b_{1}+c_{2}}$, we have

$$
\begin{gathered}
B_{1} \frac{\left|a_{2}-b_{0}-c_{1}\right|}{A_{1}}+B_{2} \frac{\left|a_{3}-c_{0}\right|}{A_{2}}+\sum_{k=3}^{\infty} B_{k} \frac{\left|a_{k+1}\right|}{A_{k}}= \\
=(1+\beta+2 \beta(1-\alpha)) \frac{\left|a_{2}-b_{0}-c_{1}\right|}{4+2 b_{1}+c_{2}}+(2(1+\beta)+2 \beta(1-\alpha)) \frac{\left|a_{3}-c_{0}\right|}{9+3 b_{1}+c_{2}}+ \\
+\sum_{k=3}^{\infty}((1+\beta) k+2 \beta(1-\alpha)) \frac{\left|a_{k+1}\right|}{(k+1)^{2}+(k+1) b_{1}+c_{2}} \leq Q .
\end{gathered}
$$

From (16), (18) and (19) we get $\sum_{k=1}^{\infty} B_{k}\left|f_{k}\right| \leq Q+\sum_{k=1}^{\infty} \eta B_{k}\left|f_{k}\right|$. Since condition (17) implies $\eta<1$, it follows that $(1-\eta) \sum_{k=1}^{\infty} B_{k}\left|f_{k}\right| \leq Q \leq 2 \beta(1-\eta)(1-\alpha)$ and, thus, condition (15) holds. By Lemma 1 function (14) is pseudoconvex in $\Pi_{0}$ of order $\alpha$ and type $\beta$.

3. Pseudoconvexity. By Lemma 2 function (14) is pseudoconvex of order $\alpha$ and type $\beta$ if $\sum_{k=1}^{\infty} k B_{k}\left|f_{k}\right| \leq 2 \beta(1-\alpha)$. As above instead (16) we have

$$
\begin{aligned}
& \sum_{k=1}^{\infty} k B_{k}\left|f_{k}\right| \leq B_{1} \frac{\left|a_{2}-b_{0}-c_{1}\right|}{A_{1}}+2 B_{2} \frac{\left|a_{3}-c_{0}\right|}{A_{2}}+\sum_{k=3}^{\infty} k B_{k} \frac{\left|a_{k+1}\right|}{A_{k}}+ \\
+ & \sum_{k=1}^{\infty}\left(\frac{(k+1) B_{k+1}}{k B_{k}} \frac{(k+1)\left|b_{0}\right|+\left|c_{1}\right|}{A_{k+1}}+\frac{(k+2) B_{k+2}}{k B_{k}} \frac{\left|c_{0}\right|}{A_{k+2}}\right) k B_{k}\left|f_{k}\right| .
\end{aligned}
$$

As above, we have $\frac{(k+1) B_{k+1}}{k B_{k}} \leq \frac{2 B_{2}}{B_{1}}$ and $\frac{(k+2) B_{k+2}}{k B_{k}} \leq \frac{3 B_{3}}{B_{1}}$ for all $k \geq 1$ and, therefore,

$$
\begin{gathered}
\frac{(k+1) B_{k+1}}{k B_{k}} \frac{(k+1)\left|b_{0}\right|+\left|c_{1}\right|}{A_{k+1}}+\frac{(k+2) B_{k+2}}{k B_{k}} \frac{\left|c_{0}\right|}{A_{k+2}} \leq \\
\leq \frac{4(1+\beta)+4 \beta(1-\alpha)}{1+\beta+2 \beta(1-\alpha)} \frac{3\left|b_{0}\right|+\left|c_{1}\right|}{9+3 b_{1}}+\frac{9(1+\beta)+6 \beta(1-\alpha)}{1+\beta+2 \beta(1-\alpha)} \frac{\left|c_{0}\right|}{16+4 b_{1}} \leq \eta^{*},
\end{gathered}
$$

where

$$
\eta^{*}=\frac{4(1+\beta)+4 \beta(1-\alpha)}{1+\beta+2 \beta(1-\alpha)} \frac{3\left|b_{0}\right|+\left|c_{1}\right|+2\left|c_{0}\right|}{9+3 b_{1}} .
$$

Finally, since $\frac{B_{2}}{A_{2}} \leq 2 \frac{B_{1}}{A_{1}}$, as above we get

$$
B_{1} \frac{\left|a_{2}-b_{0}-c_{1}\right|}{A_{1}}+2 B_{2} \frac{\left|a_{3}-c_{0}\right|}{A_{2}}+\sum_{k=3}^{\infty} k B_{k} \frac{\left|a_{k+1}\right|}{A_{k}} \leq Q^{*}
$$

where

$$
Q^{*}=\frac{1+\beta+2 \beta(1-\alpha)}{4+2 b_{1}+c_{2}}\left(\left|a_{2}-b_{0}-c_{1}\right|+4\left|a_{3}-c_{0}\right|\right)+\sum_{k=3}^{\infty} \frac{(1+\beta) k^{2}+2 \beta(1-\alpha) k}{(k+1)^{2}+(k+1) b_{1}+c_{2}}\left|a_{k+1}\right| .
$$

Using (20), (21) and (22) easy to prove the following theorem.

Theorem 2. Let $b_{1} \geq 0, c_{2} \geq 0$ and $a_{1}=1+b_{1}+c_{2}$. If $Q^{*} \leq 2 \beta\left(1-\eta^{*}\right)(1-\alpha)$ then differential equation (1) has solution (14) pseudoconvex in $\Pi_{0}$ of order $\alpha$ and type $\beta$. 
4. Growth. If $a_{1}(z)$ and $a_{0}(z) \not \equiv 0$ are entire functions then every solution of the homogeneous linear differential equation $w^{\prime \prime}+a_{1}(z) w^{\prime}+a_{0}(z) w=0$ is entire, and in order to study its growth there is preferably used the value distribution theory of memorphic functions or results of the Wiman-Valiron method (see, for example, [15, p. 114-144]). For Dirichlet series such results are obtained in [16-19].

Using formula (13) here we considerably simpler will obtain information about the growth of entire solution of differential equation (1). Suppose that $b_{1} \geq 0, c_{2} \geq 0, b_{0} \leq 0, c_{0} \leq 0$, $a_{2}-b_{0}-c_{1}>0$ and $a_{k+1}=0$ for all $k \geq k_{0} \geq 3$. Then $f_{k}>0$ for all $k \geq 1$ and (13) implies for $k \geq k_{0}$

$$
f_{k}=\frac{k\left|b_{0}\right|+\left|c_{1}\right|}{A_{k}} f_{k-1}+\frac{\left|c_{0}\right|}{A_{k}} f_{k-2}=\frac{(1+o(1))\left|b_{0}\right|}{k} f_{k-1}+\frac{(1+o(1))\left|c_{0}\right|}{k(k-1)} f_{k-2}
$$

as $k_{0} \leq k \rightarrow \infty$, whence

$$
\frac{k f_{k}}{f_{k-1}}=(1+o(1))\left|b_{0}\right|+(1+o(1))\left|c_{0}\right| \frac{f_{k-2}}{(k-1) f_{k-1}}, \quad k \rightarrow \infty .
$$

We put $A=\varlimsup_{k \rightarrow \infty} \frac{k f_{k}}{f_{k-1}}$ and $a=\varliminf_{k \rightarrow \infty} \frac{k f_{k}}{f_{k-1}}$. Then from (23) we get $A=\left|b_{0}\right|+\left|c_{0}\right| / a$ and $a=\left|b_{0}\right|+\left|c_{0}\right| / A$, i. e. $A^{2}-\left|b_{0}\right| A-\left|c_{0}\right|=0$ and $a^{2}-\left|b_{0}\right| a-\left|c_{0}\right|=0$. Thus, $A \geq 0$ and $a \geq 0$ are the roots of the quadratic equation $x^{2}-\left|b_{0}\right| x-\left|c_{0}\right|=0$ and, therefore, $A=a=$ $=\frac{1}{2}\left(\left|b_{0}\right|+\sqrt{\left|b_{0}\right|^{2}+4\left|c_{0}\right|}\right)$. Hence, it follows that $f_{k}=\frac{(1+o(1)) a f_{k-1}}{k}$ as $k \rightarrow \infty$. Therefore, for every $\varepsilon \in(0, a)$ and $k \geq k_{1}=k_{1}(\varepsilon)$

$$
\frac{(a-\varepsilon)^{k}}{k !} \leq f_{k} \leq \frac{(a-\varepsilon)^{k}}{k !}
$$

For entire Dirichlet series (2) we put $M(\sigma, F)=\sup \{|F(\sigma+i t)|: t \in \mathbb{R}\}$. The values $\varrho_{R}=\varlimsup_{\sigma \rightarrow+\infty} \frac{\ln \ln M(\sigma, F)}{\sigma}, \lambda_{R}=\varliminf_{\sigma \rightarrow+\infty} \frac{\ln \ln M(\sigma, F)}{\sigma}, T_{R}=\varlimsup_{\sigma \rightarrow+\infty} e^{-\varrho_{R} \sigma} \ln M(\sigma, F)$ and $t_{R}=$ $\underline{\lim } e^{-\varrho_{R} \sigma} \ln M(\sigma, F)$ are called $R$-order, lower $R$-order, $R$-type and lower $R$-type accordingly. Since $f_{k}>0$ for all $k \geq 1$, we have $M(\sigma, F)=F(\sigma)=O\left(e^{k_{1} \sigma}\right)+e^{\sigma} \sum_{k=k_{1}}^{\infty} f_{k} e^{k \sigma}$ as $\sigma \rightarrow+\infty$. From (24) it follows that

$$
\begin{aligned}
& O\left(e^{k_{1} \sigma}\right)+\exp \left\{(a-\varepsilon) e^{\sigma}\right\}=\sum_{k=k_{1}}^{\infty} \frac{(a-\varepsilon)^{k}}{k !} e^{k \sigma} \leq \sum_{k=k_{1}}^{\infty} f_{k} e^{k \sigma} \leq \\
& \leq \sum_{k=k_{1}}^{\infty} \frac{(a+\varepsilon)^{k}}{k !} e^{k \sigma}=O\left(e^{k_{1} \sigma}\right)+\exp \left\{\left((a+\varepsilon) e^{\sigma}\right\}, \quad \sigma \rightarrow+\infty,\right.
\end{aligned}
$$

whence $(a-\varepsilon) e^{\sigma}+o(1) \leq \ln M(\sigma, F) \leq(a+\varepsilon) e^{\sigma}+o(1)$ as $\sigma \rightarrow+\infty$, and in view of the arbitrariness of $\varepsilon$ we get $\varrho_{R}=\lambda_{R}=1$ and $T_{R}=t_{R}=a$. Thus, the following statement is proved.

Proposition 1. Let $b_{1} \geq 0, c_{2} \geq 0, b_{0} \leq 0, c_{0} \leq 0, a_{2}-b_{0}-c_{1}>0, a_{1}=1+b_{1}+c_{2}$ and $a_{k+1}=0$ for all $k \geq k_{0} \geq 3$. Then differential equation (1) has entire solution (14) such that $\ln M(\sigma, F)=\frac{1+o(1)}{2}\left(\left|b_{0}\right|+\sqrt{\left|b_{0}\right|^{2}+4\left|c_{0}\right|}\right)$ as $\sigma \rightarrow+\infty$. If $Q \leq 2 \beta(1-\eta)(1-\alpha)$ then function (14) is pseudostarlike in $\Pi_{0}$ of order $\alpha$ and type $\beta$. If $Q^{*} \leq 2 \beta\left(1-\eta^{*}\right)(1-\alpha)$ then function (14) is pseudoconvex in $\Pi_{0}$ of order $\alpha$ and type $\beta$. 


\section{REFERENCES}

1. Goluzin G.M., Geometric theory of functions of a complex variable, M.: Nauka, 1966 (in Russian). Engl. transl. Providence: AMS, 1969.

2. Kaplan W., Close-to-convex schlicht functions, Michigan Math. J., 1 (1952), №2, 169-185.

3. Shah S.M., Univalence of a function $f$ and its successive derivatives when $f$ satisfies a differential equation, II, J. Math. anal. and appl., 142 (1989), 422-430.

4. Sheremeta Z.M., Close-to-convexity of entire solutions of a differential equation, Matem. Metody ta Fiz.-Mekh. Polya, 42 (1999), №3, 31-35 (in Ukrainian).

5. Sheremeta Z.M., The properties of entire solutions of one differential equation, Diff. Equ., 36 (2000), №8, 1155-1161. doi: 10.1007/BF02754183

6. Sheremeta Z.M., On entire solutions of a differential equation, Mat. Stud., 14 (2000), №1, 54-58.

7. Sheremeta Z.M., On the close-to-convexity of entire solutions of a differential equation, Visnyk Lviv Univ. Series Mech. Math., 58 (2000), 98-106 (in Ukrainian).

8. Sheremeta Z.M., Sheremeta M.N., Closeness to convexity for entire solutions of a differential equation, Diff. Equ., 38 (2002), №4, 496-501. doi: 10.1023/A:1016355531151

9. Sheremeta Z.M., Sheremeta M.M., Convexity of entire solutions of a differential equations, Matem. Metody ta Fiz.-Mekh. Polya, 47 (2004), №2, 186-191 (in Ukrainian).

10. Trukhan Yu., Sheremeta M., Closeness-to-convexity of solutions of a second order nonhomogeneous differential equation, Visnyk Lviv Univ. Series Mech. Math., 88, (2019), 98-106. http://dx.doi.org/10.30970/vmm.2019.88.098-106 (in Ukrainian)

11. Sheremeta M.M., Trukhan Yu.S. Properties of analytic solutions of a differential equation, Mat. Stud., 52 (2019), №2, 138-143. doi: 10.30970/ms.52.2.138-143

12. Holovata O.M., Mulyava O.M., Sheremeta M.M., Pseudostarlike, pseudoconvex, and close-topseudoconvex Dirichlet series satisfying differential equations with exponential coefficients, J. Math. Sci. (United States), 249 (2020), №3, 369-388. doi: 10.1007/s10958-020-04948-1

13. Sheremeta M.M., Geometric properties of analytic solutions of differential equations. Lviv: Publisher I.E. Chyzhykov, 2019.

14. Sheremeta M.M., Pseudostarlike and pseudoconvex Dirichlet series of the order $\alpha$ and the type $\beta$, Mat. Stud., 54 (2020), №1, 23-31. doi: https://doi.org/10.30970/ms.54.1.23-31

15. Wittich H., Neuere Untersuchungen uber eindeutige analytische Functionen, Springer-Verlag, Berlin, 1955.

16. Sheremeta M.N., Asymptotic properties of entire functions defined by Dirichlet series and of their derivatives, Ukr. Math. J., 31 (1979), №6, 558-564. doi: 10.1007/BF01092538

17. Sheremeta M.N., On the derivative of an entire Dirichlet series, Mathematics of the USSR - Sbornik, 65 (1990), №1, 133-145. doi: 10.1070/SM1990v065n01ABEH002076

18. Salo T.M., Skaskiv O.B., Stasyuk Ya.Z., On a central exponent of entire Dirichlet series, Mat. Stud., 19 (2003), №1, 61-72.

19. Skaskiv O.B., Stasyuk Ya.Z., On the Wiman theorem for absolutely convergent Dirichlet series, Mat. Stud., 20 (2003), №2, 133-142.

Ivan Franko National University of Lviv, Ukraine m.m.sheremeta@gmail.com 